\title{
Large Sternal Exostoses presenting as stridor: A Surgical and Anesthetic challenge
}

\author{
Alok Sharma ${ }^{1}$, Surendra Patel ${ }^{1}$, Danishwar Meena ${ }^{1}$, Ranjit Sahu ${ }^{1}$, Amit Goyal ${ }^{1}$, Pawan \\ $\operatorname{Garg}^{1}$, and Mritunjay Kumar ${ }^{1}$
}

${ }^{1}$ All India Institute of Medical Sciences Jodphur

May 29, 2020

\begin{abstract}
Large osteochondroma arising from chest wall and sternum is uncommon and presentation with airway compression is further uncommon. Here we present a case of large chest wall osteochondroma as a part of Hereditary multiple exostoses in a 9 years old boy presented with a history of stridor and shortness of breath. The bony mass of the right chest wall was extending up to a suprasternal notch and compressing the trachea. The case was successfully managed by initial femoro-femoral cardiopulmonary bypass under local anesthesia prior to the induction of anesthesia to prevent respiratory collapse, followed by debulking surgery was done.
\end{abstract}

\section{Introduction}

Hereditary multiple exostoses (HME) is an autosomal dominant disease characterized by abnormal growth of bones, mainly involving epiphyses of long bones. In HME involvement of Sternum is very rare ${ }^{1}$. Large sternal mass compressing airway needs to be managed by a multidisciplinary approach to prevent injury to great vessels, heart, and risk of fatal respiratory failure during induction of anesthesia. We are reporting this case due to a very rare presentation of a large sternal exostoses compressing airways, which was managed successfully.

\section{Case report}

A 9 years old male child presented with complaints of rapidly progressive shortness of breath for the last 2 months more on supine position. The patient had a history of progressively increasing swellings in upper limbs, lower limbs, and chest wall since the age of 2 years. The patient had a large, irregular, bony-hard, swelling involving Upper $2 / 3$ of sternum, suprasternal notch and right shoulder. [fig.1]. The bony mass over suprasternal notch was compressing the cervical part of the trachea, causing stridor. The swellings of extremities were of cosmetic concerns only. The father of the patient also had bony swellings over the right humerus and left femoral bone. Computed tomography (CT) of the thorax showed a large exophytic bony mass involving sternum and right side of chest wall [Fig $2 \mathrm{~A}, 2 \mathrm{~B}, 2 \mathrm{C}, 3 \mathrm{~A}$, and 3B]. The mass was encroaching in suprasternal notch causing compression of the trachea at the level of lower border of C7 vertebra. The minimum anteroposterior diameter of the trachea was $3.4 \mathrm{~mm}$ [Fig. 2D]. Multiple other bony exostoses also have seen arising from ribs [Fig 2C] and scapula.

The key challenge in this patient was the severe external compression of the trachea, not permitting the minimum required size of the endotracheal tube. The case was discussed by the multidisciplinary team. Considering the above circumstances, it was decided to perform femoro-femoral Cardiopulmonary Bypass (CPB) under local anesthesia preceding the induction of general anesthesia (GA). Various invasive lines were placed under local anesthesia (LA) for intraoperative monitoring after verbally explaining to the patient. The right femoral artery and vein were exposed under LA and were canulated with 16 and 20 Fr sized canula 
(Edwards FemFlex@) respectively. Cardiopulmonary bypass (CPB) was established at $80 \%$ flow. After that Anaesthesiologists induced the patient and put Flexo-metallic endotracheal tube (ET tube) $4 \mathrm{~mm}$ size using a fiber-optic laryngoscope and later, it was exchanged with $5.5 \mathrm{~mm}$ using a tube-exchanger. After confirmation of good ventilation via the ET tube, $\mathrm{CPB}$ was weaned. The total CPB time was 85 minutes.

A ' $T$ ' shaped incision was given over swelling with transverse limb at the level of the thyroid cartilage and the vertical limb extending up to xiphisternum. The bony-mass was removed in piecemeal by using chisel, hammer, and oscillating saw while taking care of major neck vessels and trachea. While the suprasternal extension of mass was completely removed, only debulking of sternal body and chest wall mass were done to achieve fair cosmetics result. No evidence of tracheomalacia was found. In view of uneventful post-operative recovery, and absence of any residual respiratory difficulty, the patient was discharged on the fourth postoperative day. Histopathology report was suggestive of Osteochondroma. CT thorax done at one-month follow-up showed an adequate debulking of mass [Fig 3C, 3D] with the normal trachea. The patient was asymptomatic and aesthetically satisfied after 1 year.

\section{Discussion}

Osteochondroma (Exostoses) is the commonest benign bone tumor ${ }^{2}$. These are cartilage-capped bony projections arising on the external surface of the bone and containing a marrow cavity that is continuous with that of the underlying bone ${ }^{3}$. The majority of lesions are solitary, and around $15 \%$ are multiple as a part of Hereditary multiple exostoses (HME) syndrome. The patient usually presents in childhood with bony swelling in limbs, causing deformity, and sometimes symptoms related to compression of surrounding structures. Around $50 \%$ of patients present by the age of 5 years and $80 \%$ by 10 years of age ${ }^{1}$. Our patient had multiple large swelling over upper limbs, lower limbs, and chest wall since the age of 2 years, which were gradually increasing in size. He presented with difficulty in breathing and stridor on supine position due to compression of trachea by large suprasternal exostoses mass. The patient's father also had similar bony swellings over limbs, which supports our diagnosis of Hereditary multiple exostoses (HME).

The use of extracorporeal circulation in management of airway obstruction was first reported by Onozawa et al in 1999, in a patient with thyroid carcinoma ${ }^{4}$. The use of CPB before induction of GA for surgeries of large neck or mediastinal masses compressing airways has been reported ${ }^{5,6}$. With the induction of GA, there is a loss of respiratory muscle tone and decrease functional residual capacity which in patients with already compromised airways may lead to respiratory collapse and death ${ }^{6}$. The tip of the ET tube can injure our patient's trachea at the site of maximal compression leading to catastrophe.

We have managed our case successfully while avoiding catastrophic events by instituting femoro-femoral CBP under LA preceding to the induction of GA.

\section{Conclusion}

In patients of compromised airways due to compression by surrounding tumors/ masses, femoro-femoral cardiopulmonary bypass is a safe method to prevent further airway compromise while induction of anesthesia.

\section{References}

1. Ryckx A, Somers JF, Allaert L. Hereditary multiple exostosis.Acta Orthop Belg. 2013;79(6):597-607.

2. Murphey MD, Choi JJ, Kransdorf MJ et al. Imaging of osteochondroma: variants and complications with radiologic-pathologic correlation.Radiographics. 2000;20:1407-1434.

3. Khurana J, Abdul-Karim F, and Bovée JVMG: Osteochondroma. In: Fletcher CDM, Unni KK, and Mertens F, eds. World Health Organization Classification of Tumours. Pathology and Genetics of Tumours of Soft Tissue and Bone. Lyon, France: IARC; 2002:234-236

4. Onozawa H, Tanaka T, Takinami M, Kagaya S, Tanifuji Y. Anesthetic management using extracorporeal circulation of a patient with severe tracheal stenosis by thyroid cancer (Japanese). Masui.1999;48(6):658-61

5. Tempe DK, Arya R, Dubey S, Khanna S, Tomar AS, Grover V, Nigam M, Makwane UK. Mediastinal 
mass resection: femorofemoral cardiopulmonary bypass before induction of anesthesia in the management of airway obstruction. J Cardiothorac Vasc Anesth. 2001;15:233-6.

6. Kim CW, Kim DH, Son BS, et al. The Feasibility of Extracorporeal Membrane Oxygenation in the Variant Airway Problems. Ann Thorac Cardiovasc Surg . 2015; 21(6):517-522.

\section{Figure captions}

Figure. 1: A large, irregular, swelling involving Upper 2/3 of sternum extending to suprasternal notch (blue arrow) and right shoulder.

Figure 2: Computed tomography (CT) of the thorax in axial (A) (B) and coronal (C) and Sagittal (D) plane, showing large exophytic bony mass (black Asterisk) arising from the right side of the chest wall. The mass is causing compression over the airway resulting in severe narrowing of the trachea (minimal diameter of 3.4 $\mathrm{mm}$ ) (white arrow). Multiple other bony exostoses arising from ribs (dashed arrow).

Figure 3: Volume rendered (VR) 3D images of the chest in coronal (A) and oblique plane (B), showing large bony mass (Asterisk) arising from the right side of the chest wall causing significant deformity. Multiple other bony exostoses arising from Ribs and scapula (white arrow). Postoperative 3D VR images of the chest in coronal $(\mathrm{C})$ and oblique plane (D) showing a significant reduction in the size of the bony mass.

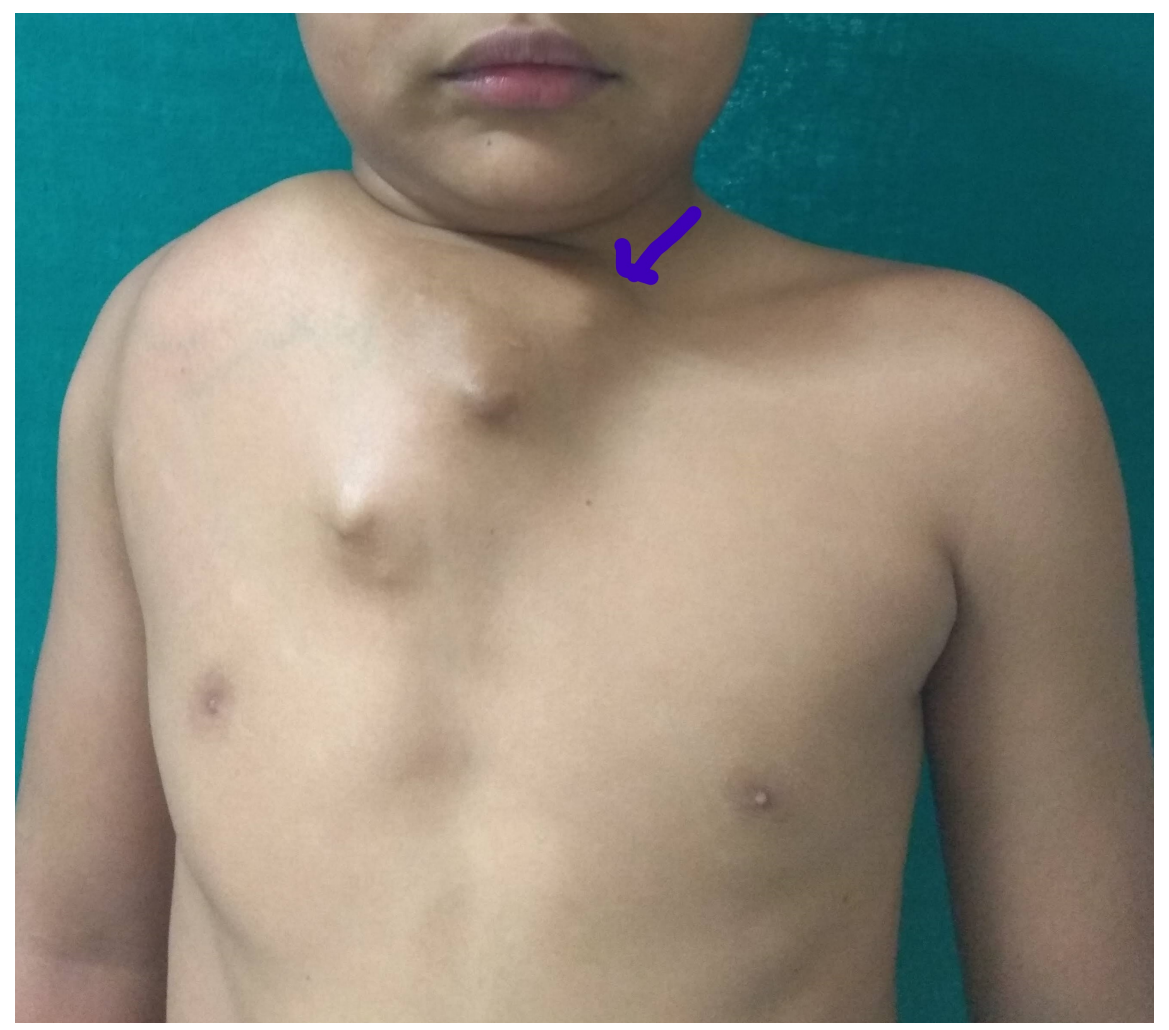



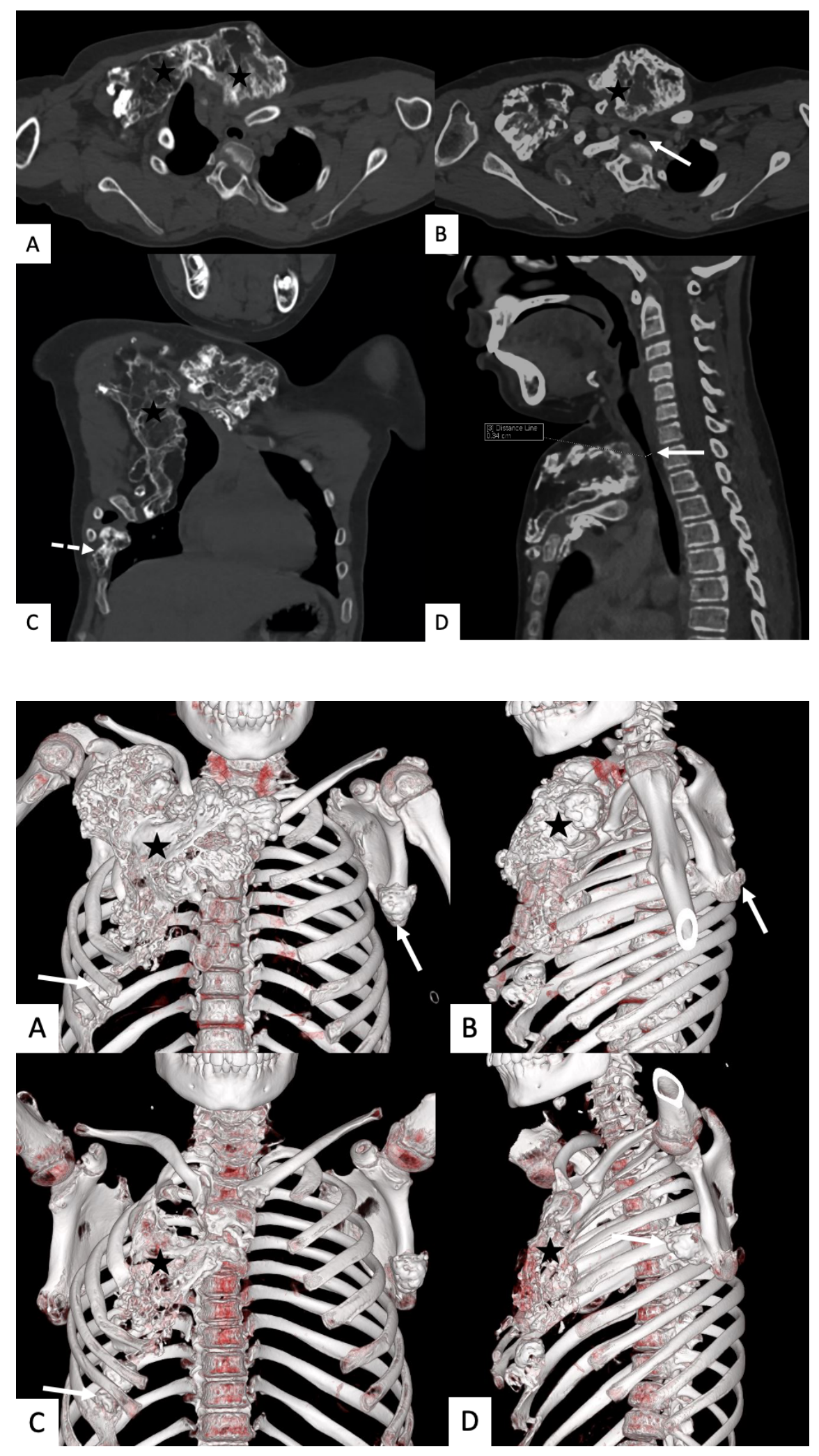